\title{
Analysis of 12 Chinese Patients with Proline-to-Leucine Mutation at Codon 102-Associated Gerstmann-Sträussler-Scheinker Disease
}

\author{
Jing Wang ${ }^{\mathrm{a}}$ \\ Kang Xiao ${ }^{a}$ \\ Wei Zhou ${ }^{a}$ \\ Qi Shi ${ }^{\mathrm{a}}$ \\ Xiao-Ping Dong ${ }^{\mathrm{a}, \mathrm{b}}$ \\ aState Key Laboratory for Infectious \\ Disease Prevention and Control, \\ Collaborative Innovation Center \\ for Diagnosis and Treatment \\ of Infectious Diseases \\ (Zhejiang University), National Institute \\ for Viral Disease Control and Prevention, \\ Chinese Center for Disease Control and \\ Prevention, Beijing, China \\ ${ }^{b}$ Center of Global Public Health, \\ Chinese Center for Disease Control and \\ Prevention, Beijing, China
}

\begin{abstract}
Background and Purpose Gerstmann-Sträussler-Scheinker disease (GSS) with a prolineto-leucine mutation at codon $102(\mathrm{P} 102 \mathrm{~L})$ in the PRNP gene is the most frequently reported GSS subtype worldwide. This study aimed to determine the epidemiological, clinical, genetic, and laboratory characteristics of 12 Chinese patients with P102L-associated GSS (henceforth P102L GSS)

Methods The enrolled P102L GSS cases were analyzed according to the diagnostic criteria for Creutzfeldt-Jakob disease (CJD) issued by the China National Health Commission.

Results The median onset age was 50 years (range 34 to 67 years) and sex ratio was 1:2 (males:females). Most patients displayed more than one foremost symptom. Movement symptoms were frequently reported ( 9 of the 12 cases), followed by rapidly progressing dementia ( 7 cases), mental problems (5 cases), and slowly progressing dementia (2 cases). Almost all cases displayed more sporadic CJD (sCJD)-associated neurological symptoms and signs as time progressed. Five (45.5\%) of 11 cases were cerebrospinal fluid 14-3-3 positive, and 2 (25\%) of 8 cases exhibited periodic sharp wave complexes in electroencephalograms. MRI abnormalities were detected in all 11 of the scanned patients. Methionine homozygous genotype at codon 129 (M129M) and glutamic acid homozygous at codon 219 (E219E) homozygosity was present in 11 cases, while 1 case was M129M homozygous and glutamic acid/lysine heterozygous at codon 219 (E219K) heterozygous. Ten of the 12 cases recalled a disease-related family history during the clinical interviews. The median survival from symptom onset of the seven dead cases was 16 months (range 10 to 44 months). Patients showing the sCJD phenotype (rapidly progressing dementia) appeared to be associated with a shorter survival time.

Conclusions The indistinguishable clinical features of P102L GSS patients with sCJD, especially in the early stage, support the importance of PRNP testing for diagnosing GSS.

Key Words prions, Gerstmann-Sträussler-Scheinker disease, PRNP proline-to-leucine mutation at codon 102 mutation.
\end{abstract}

Received August 2, 2018

Revised November 20, 2018

Accepted November 21, 2018

\section{Correspondence}

Xiao-Ping Dong, $\mathrm{MD}, \mathrm{PhD}$

State Key Laboratory for Infectious

Disease Prevention and Control,

Collaborative Innovation Center

for Diagnosis and Treatment

of Infectious Diseases

(Zhejiang University), National Institute

for Viral Disease Control and

Prevention, Chinese Center

for Disease Control and Prevention,

Chang-Bai Rd 155, Beijing 102206,

China

Tel +86-10-58900815

Fax +86-10-58900815

E-mail dongxp238@sina.com

\section{INTRODUCTION}

Autosomal-dominant mutations in the prion protein gene, $P R N P$, lead to genetic prion diseases that account for $10-15 \%$ of human prion diseases. ${ }^{1,2}$ Due to differences in clinical and neuropathological features, human genetic prion diseases are categorized into three groups: genetic Creutzfeldt-Jakob disease (CJD), Gerstmann-Sträussler-Scheinker disease (GSS), and fatal familial insomnia. ${ }^{1}$

The history of GSS dates back to 1936, when an Austrian family with GSS was reported by J. Gerstmann, E. Sträussler, and I. Scheinker., ${ }^{3,4}$ In 1995, a proline-to-leucine mutation at codon 102 (P102L) in the PRNP gene was identified in this family. ${ }^{4}$ More than 16 different

( This is an Open Access article distributed under the terms of the Creative Commons Attribution Non-Commercial License (https://creativecommons.org/licenses/by-nc/4.0) which permits unrestricted non-commercial use, distribution, and reproduction in any medium, provided the original work is properly cited. 
point mutations (including P84S, P102L, P105L, P105S, A117V, G131V, S132I, V176G, H187R, F198S, D202N, E211D, Q212P, Q217R, Y218N, and M232T) and several octapeptide repeat insertions in PRNP are now linked to GSS, with P102L reportedly to be the most common worldwide. ${ }^{5-10}$ P102L-associated GSS (henceforth P102L GSS) was also the most commonly identified GSS in a Chinese population. $^{11}$

The most-common phenotype of GSS patients is slowly progressing cerebella ataxia initially, with parkinsonism and dementia appearing later in the clinical course. ${ }^{12,13}$ The presence of multicentric prion protein amyloid plaques in neuropathology remains the key feature of GSS that differentiates it from most of the other genetic prion diseases., ${ }^{3,14}$ The survival times of GSS patients reportedly vary from 1 to 10 years worldwide. ${ }^{3}$ However, some patients with symptoms more typical of sporadic CJD (sCJD) have a much shorter clinical course. ${ }^{15-17}$

There have been 12 P102L GSS cases diagnosed by the Chinese National Surveillance Network for CJD under the framework of the Chinese Center for Disease Control and Prevention (CCDC) since 2006, constituting about $8 \%$ of all diagnosed genetic prion diseases. Here we investigated the epidemiological, clinical, genetic, and laboratory characteristics of these P102L GSS patients.

\section{METHODS}

\section{Clinical data collection and case identification}

Twelve Chinese P102L GSS cases were enrolled in this study. Information was obtained from the Chinese National Surveillance Network for CJD under the leadership of the CCDC as described previously. ${ }^{18}$ Briefly, the appearance of periodic sharp wave complexes (PSWC) was regarded as a prion-disease-specific EEG abnormality. MRI findings were considered to be abnormal in the presence of high signal intensities in the caudate/putamen and/or symmetrical or dissymmetrical cortical ribbon syndrome in diffusion-weighted imaging (DWI). The geographical distribution was determined based on the registered permanent addresses of the patients. The survival time was calculated as the duration from disease onset to death (analyzed from January 1, 2005 to April 2, 2018). The final diagnosis was made according to the diagnostic criteria for CJD issued by the China National Health Commission. ${ }^{19}$

\section{Laboratory tests}

Blood and CSF were collected by physicians in local hospitals and transferred to the center. Western blotting of CSF for 14-3-3 protein and PRNP sequencing were conducted following the standard operating procedure of the Chinese Na- tional Surveillance Network for CJD. ${ }^{18,20}$ In brief, a $20-\mu \mathrm{L}$ CSF sample was separated in $12 \%$ sodium dodecyl-sulfate polyacrylamide gel electrophoresis and immunoblotted with a 14-3-3-specific monoclonal antibody (1:500 diluted; SC133233, Santa Cruz; https://www.scbt.com/scbt/product/ pan-14-3-3-antibody-b-8). The genomic DNA of the patients was extracted from peripheral blood samples for sequencing analysis of PRNP and the polymorphisms of codons 129 and 219. All experiments in which mutations were identified by comparisons with the standard (NCBI: NM-183079.1) were repeated at least once using new blood samples in order to avoid misreading.

\section{Statistical analyses}

The statistical analyses were performed using SPSS 20.0 statistical software (IBM Corp., Armonk, NY, USA). Categorical variables were compared using Fisher's exact test, while the relationship between disease duration and the speed of dementia progression was analyzed using logistic regression. Probability $(p)$ values less than 0.05 were considered to be statistically significant. Survival analyses were performed using GraphPad Prism software (GraphPad Software, San Diego, CA, USA).

\section{Ethics statement}

The study was approved by the Research Ethics Committee of National Institute for Viral Disease Control and Prevention, CCDC (approval no. \#2013031). Written informed content was obtained from a family member or relative of each patient in accordance with the requirements of the Chinese National Surveillance Network for CJD.

\section{RESULTS}

\section{General information}

According to the data of the Chinese National Surveillance Network for CJD, which started officially in 2006, 12 P102L GSS patients were identified and diagnosed by April 2018, all of whom were Han Chinese. Based on their permanent addresses, most of the patients came from the eastern part of China: three from Shandong province, and one from each of Shanxi, Henan, Hebei, Jiangxi, Anhui, Hubei, Jiangsu, Zhejiang, and Jilin. Clinical interviews did not reveal any blood relationship among these patients. There were four males and eight females, giving a sex ratio of 1:2. The median onset age was 50 years, ranging from 34 to 67 years. Most of the patients were aged $40-49$ years $(n=5)$, followed by $50-59$ years $(n=3)$ and 60-69 years $(n=3)$ (Fig. 1). 


\section{Clinical features}

Most of the patients presented with more than one symptom at the onset, which included movement symptoms (gait and walking instability), mental problems (e.g., anxiety, dystrophy, irritability, and emotional lability), rapid or slow progression of memory decline, and dementia. As indicated in Table 1, movement symptoms were reported in $75 \%$ of the cases $(n=9)$. The proportion of movement problems was slightly higher for an onset age of $<50$ years $(5 / 6,83.3 \%)$ than for one of $\geq 50$ years $(4 / 6,66.7 \%)$. Rapidly progressing dementia was reported in 7 cases (58.3\%). Mental problems such as anxiety and irritability appeared in 5 cases (41.7\%), and were more common for an onset age of $\geq 50$ years $(4 / 6$, $66.7 \%)$ than for one of $<50$ years $(1 / 6,16.7 \%)$. Additionally,

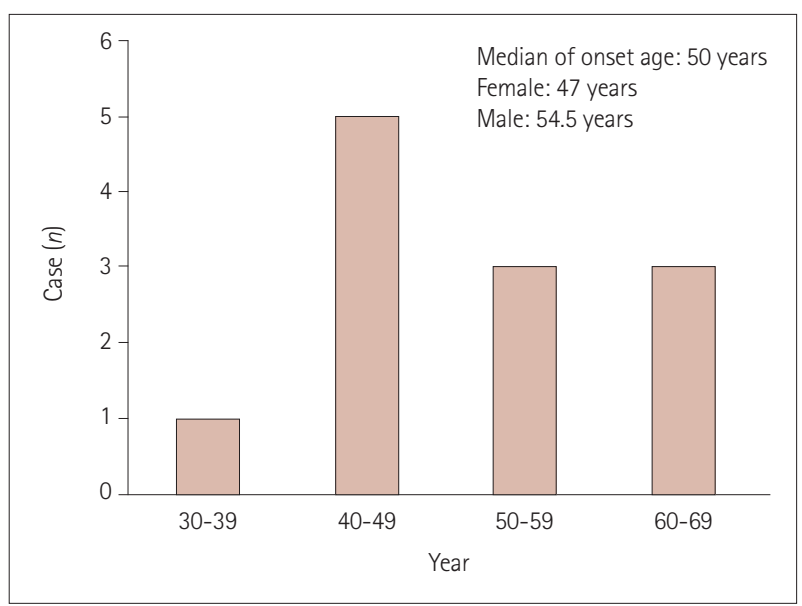

Fig. 1. Distribution of the onset ages of 12 Chinese P102L Gerstmann-Sträussler-Scheinker disease patients. The median onset ages overall and in the different groups are shown at the top right. there were 2 cases of slowly progressing dementia, and 4 cases with a medical history of high blood pressure lasting for 1-4 years based on the normal values of blood pressure for various ages of Chinese subjects.

Along with the disease progression, more sCJD-associated neurological symptoms and signs appeared. Progressive dementia was recorded in all cases (12/12,100\%). Pyramidal and extrapyramidal symptoms $(10 / 12,83.3 \%)$, myoclonus $(8 / 12$, $66.7 \%)$, and visual or cerebellar problems $(6 / 12,50 \%)$ also appeared frequently. Akinetic mutism was observed in the late stage of disease in 11 cases (Supplementary Table 1 in the online-only Data Supplement). No statistical differences were found in the comparisons between the symptoms and the groups of gender or onset age.

\section{Clinical examination and laboratory tests}

EEG was applied to 8 cases at least once during hospitalization, two of which exhibited PSWC. Eleven of the 12 cases received MRI scanning, which revealed SCJD-specific abnormalities in all cases. Three cases had high signal intensities in the caudate/putamen, 3 cases had DWI ribbon-like signals, and 5 cases had both abnormalities (Supplementary Table 1 in the online-only Data Supplement). There were no statistically significant differences in the distributions of specific EEG and MRI abnormalities between the groups of gender and onset age. Lumbar puncture was performed in $11 \mathrm{pa}-$ tients, and the routine biochemistry values were all within the normal ranges. Western blotting for 14-3-3 protein revealed positive bands in the CSF samples from 5 cases (45.5\%) (Table 1).

Table 1. The main clinical and laboratory features of 12 Chinese patients with P102L Gerstmann-Sträussler-Scheinker disease

\begin{tabular}{|c|c|c|c|c|c|c|c|}
\hline \multirow{2}{*}{ Clinical feature } & \multirow{2}{*}{$\begin{array}{c}\text { Total } \\
(n=12)\end{array}$} & \multicolumn{3}{|c|}{ Age at onset (years) } & \multicolumn{3}{|c|}{ Sex } \\
\hline & & $<50(n=6)$ & $\geq 50(n=6)$ & $p$ & Male $(n=4)$ & Female $(n=8)$ & $p$ \\
\hline Age at onset, years & $50[34-67]$ & - & - & - & $54.5[48-67]$ & 47 [34-67] & 0.389 \\
\hline \multicolumn{8}{|l|}{ Onset symptoms } \\
\hline Movement symptoms & $9(75.0)$ & $5(83.3)$ & $4(66.7)$ & 0.500 & $4(100)$ & $5(62.5)$ & 0.745 \\
\hline Rapidly progressing dementia & $7(58.3)$ & $4(66.7)$ & $3(50.0)$ & 0.500 & $2(50.0)$ & $5(62.5)$ & 0.576 \\
\hline Mental problems & $5(41.7)$ & $1(16.7)$ & $4(66.7)$ & 0.121 & $3(75.0)$ & $2(25.0)$ & 0.576 \\
\hline Slowly progressing dementia & $2(16.7)$ & 0 & $2(33.3)$ & 0.576 & $1(25.0)$ & $1(12.5)$ & 0.227 \\
\hline \multicolumn{8}{|c|}{ Symptoms following disease progression } \\
\hline Progressive dementia & $12(100.0)$ & $6(100.0)$ & $6(100.0)$ & - & $4(100.0)$ & $8(100.0)$ & - \\
\hline Visual/cerebellar problems & $6(50.0)$ & $4(66.7)$ & $2(33.3)$ & 0.284 & $3(75.0)$ & $3(37.5)$ & 0.273 \\
\hline Myoclonus & $8(66.7)$ & $5(83.3)$ & $3(50.0)$ & 0.273 & $3(75.0)$ & $5(62.5)$ & 0.594 \\
\hline Pyramidal/extrapyramidal feature & 10 (83.3) & $5(83.3)$ & $5(83.3)$ & 0.773 & $4(100)$ & $6(75.0)$ & 0.424 \\
\hline High blood pressure & $4(33.3)$ & $2(33.3)$ & $2(33.3)$ & 0.727 & $2(50.0)$ & $2(25.0)$ & 0.406 \\
\hline CSF 14-3-3 positivity & $5(45.5)^{*}$ & $3(60.0)^{*}$ & $2(33.3)$ & 0.392 & $1(25.0)$ & $4(57.1)^{*}$ & 0.348 \\
\hline MRI abnormality & $11(100.0)^{+}$ & $5(100.0)^{+}$ & $6(100.0)$ & - & $4(100.0)$ & $7(100.0)^{+}$ & - \\
\hline
\end{tabular}

Data are $n(\%)$ or median [range] values.

${ }^{*}$ No CSF sample for one case in the group, ${ }^{+} \mathrm{MRI}$ scanning not performed for one case in the group. 


\section{Survival time}

Up to April 2018, 7 cases had died on a known date, 4 were still alive, and one was lost to follow-up. The survival times based on different sex and onset ages are shown in Fig. 2. The clinical course of the 7 dead cases varied from 10 to 44 months, with a median of 16 months. Five of the 7 cases (71.4\%) with a short survival time (less than 2 years) complained of rapidly progressing dementia at the onset, while the other two patients with a long duration (longer than 40 months) displayed relatively slowly progressing dementia. Moreover, female and younger patients at onset seemed to show shorter survival times than males and older-onset patients (Fig. 2). However, no statistical significance was found, which was possibly due to the small number of cases.

\section{$P R N P$ sequencing and family history}

PRNP sequencing confirmed that all 12 cases were carrying the P102L mutation in 1 PRNP allele. All cases had the methionine homozygous genotype at codon 129 (M129M), while 10 cases were glutamic acid homozygous at codon 219 (E219E) and one was glutamic acid/lysine heterozygous at codon 219 (E219K) (Supplementary Table 1 in the online-only Data Supplement). No other mutation was noted in the remaining PRNP sequences analyzed.

Carefully tracing of the disease-related family histories identified that the parent and/or sibling of 10 cases had died

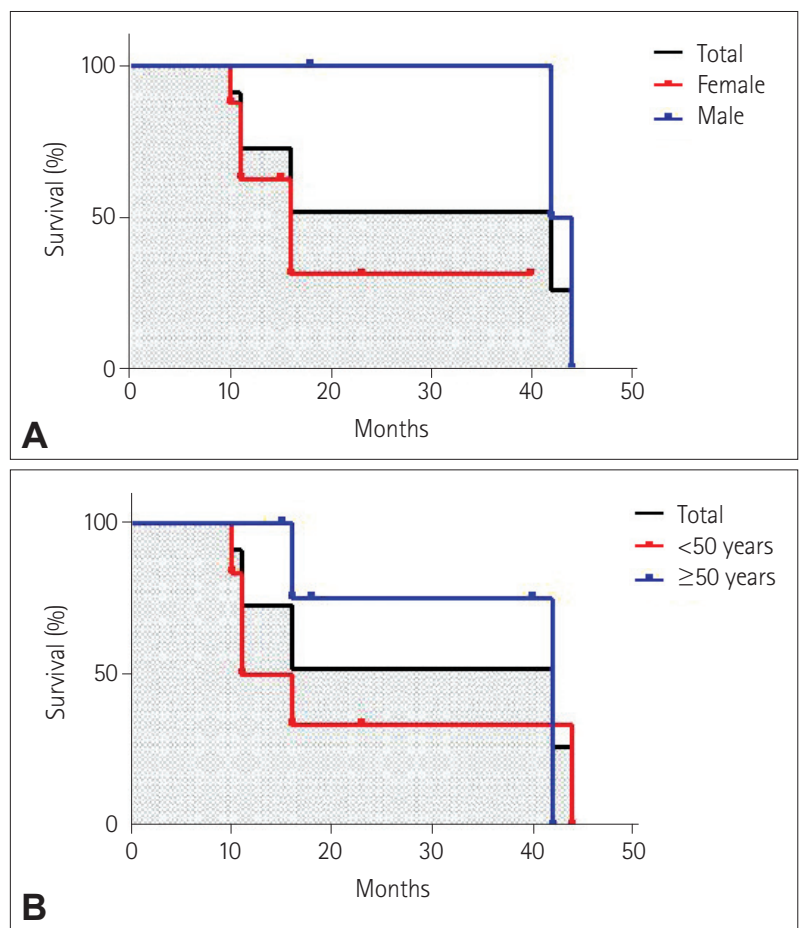

Fig. 2. Survival times of 12 P102L Gerstmann-Sträussler-Scheinker disease patients according to gender (males and females, A) and onset age $(<50$ years or $\geq 50$ years, $B)$. of similar neurological problems without a definite diagnosis. Only 2 cases (Cases 3 and 10) denied having a relevant family history. As shown in Fig. 3, Cases 6, 7, and 8 recalled similar symptoms only in the generation of their parents, while in Case 1 this was only in the proband generation. Cases 2, 5,11 , and 12 had other close family members who had died with neurological symptoms both in their parent and proband generations. Cases 3 and 4 reported a disease-related family history during hospitalization, but their family members refused to provide detailed information. Only four family members from two families donated blood samples for PRNP sequencing: three from Case 9 who denied having a diseaserelated family history, and one from Case 6. PRNP sequencing verified that the mother and son of Case 9 had a wild-type PRNP genotype, while her daughter was a P102L missense mutation carrier. The son of Case 6 also carried the P102L mutation. Both of these two P102L mutation carriers were M129M and E219E homozygous, and had not exhibited any neurological abnormality up to April 2018.

\section{DISCUSSION}

This study systematically evaluated the epidemiology, clinical, genetic, and laboratory features of 12 Chinese P102L GSS patients. There were more female than male patients, but neither the clinical nor laboratory examination revealed any sex-associated difference. Like the P102L GSS cases reported worldwide, ${ }^{2,3}$ the onset ages for the present Chinese patients were relatively young, with a median of 50 years. However, the average disease duration of the seven Chinese patients seems to be shorter than the global value (52 months, ranging from 7 to 132 months). ${ }^{10,16,21}$ Patients showing the sCJD phenotype (rapidly progressing dementia) seem to have a shorter survival time (10-16 months). It is particularly notable that patients with a prominent psychiatric onset usually have an early onset and death. ${ }^{2}$ The youngest Chinese P102L GSS patient in the present study, a 34-year-old woman with a psychiatric family history, also had a short survival time (11 months) and had mental syndrome as her initial symptoms. Whether and why Chinese GSS patients have relatively short survival needs to be investigated in more cases.

With regard to other symptoms, cognitive decline is generally mild in P102L GSS cases and this aggravates during disease progression. ${ }^{21}$ Three-quarters of the present Chinese patients had early cognitive deficits, and the progression of dementia was relatively rapid in most of them, showing an sCJDlike phenotype. Among 57 Japanese P102L GSS cases, 21\% presented with an SCJD-like phenotype with early and prominent dementia. ${ }^{17}$ Another study found that $40 \%$ of cases showed cognitive symptoms at the onset. ${ }^{10}$ Whether the higher 


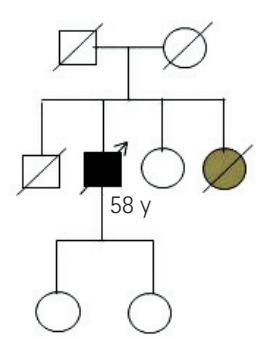

Case 1
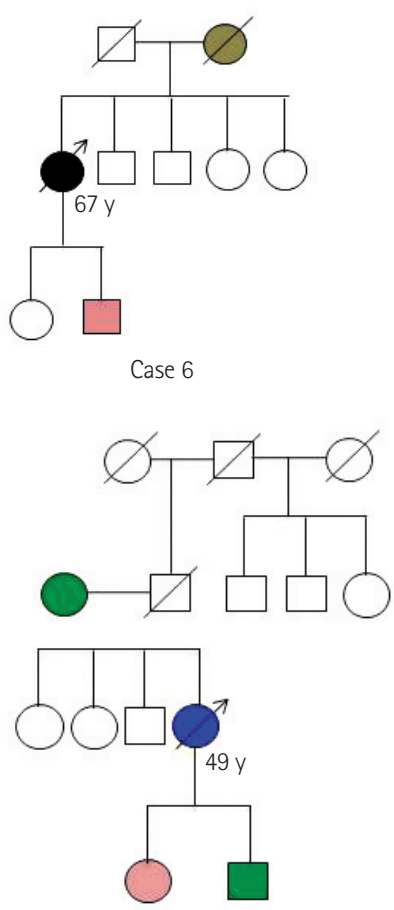

Case 9

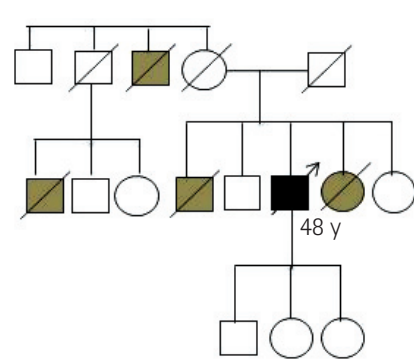

Case 2

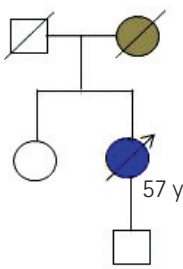

Case 7

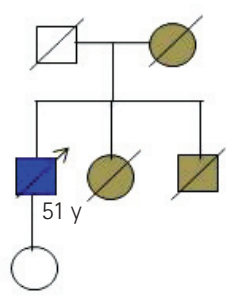

Case 11

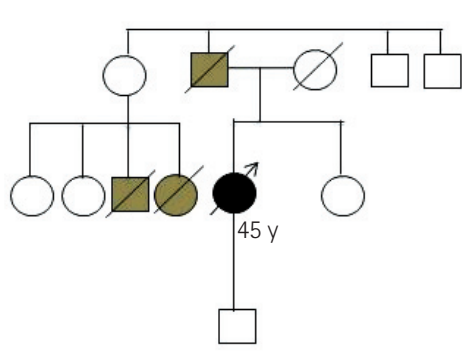

Case 5

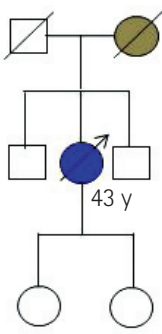

Case 8

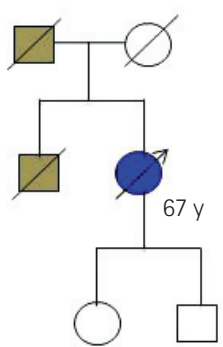

Case 12

Fig. 3. Disease-related family histories and P102L mutations in PRNP in several families. The shapes, lines, and colors are defined in the panel below the figure. P102L: proline-to-leucine mutation at codon 102.

proportion of cognitive problems is a characteristic of Chinese P102 GSS patients needs to be investigated further in more cases.

The sCJD-associated abnormalities in MRI appear frequently in Chinese cases of P102L GSS, especially in the relatively late stage. High signal intensities in the caudate/putamen and DWI ribbon-like signals are equally observable. A high proportion (80\%) of MRI abnormalities was found in Japanese GSS patients with a rapidly progressing clinical phenotype.${ }^{17}$ However, a investigation based on data obtained in the EuroCJD study found fluid-attenuated inversion recovery or DWI hyperintensities in the basal ganglia in 30\% of the P102L GSS cases. ${ }^{16}$ Other studies have even noted cortical ribboning or deep nuclei hyperintensities to be very uncommon. ${ }^{22}$ The significance of MRI findings in P102L GSS therefore needs further evaluation. Only one-quarter and less than half of the Chinese patients in the present study were verified as having PSWC in EEG and positivity for 14-3-3 protein 
in the CSF, respectively. Coincidental, PSWC in EEG and 14-3-3 positivity in the CSF were observed in 50\% and 31\% of Caucasian GSS patients, respectively. ${ }^{23}$ It therefore appears that diagnosing P102L GSS based on EEG and CSF 14-3-3 positivity will result in fewer cases than when applying MRI.

The disease-associated family history of P102L GSS patients seems to vary greatly. The EuroCJD study involved 52 cases in European countries and found a family history in $69.7 \%$ of them. ${ }^{23}$ However, other studies involving German and Japanese patients found positive family histories in $84 \%$ to $100 \%$ of cases. ${ }^{16,17}$ A high positivity rate (83.3\%) for the family history was found in the present Chinese P102L GSS patients. It should be noted that the disease-related family histories for the patients in this study were obtained not only from routine clinical interviews performed during hospitalization but also during the subsequent surveillance followup. Therefore, careful and repeated interviews with patients and family members are highly recommended.

Polymorphism of PRNP is associated with both the susceptibility and phenotype of prion diseases. ${ }^{24,25}$ The polymorphism at PRNP codon 219 with the substitution of glutamate for lysine seems to be found only in Asian population, and not in Caucasians. ${ }^{26,27}$ P102L GSS patients with this polymorphism have been described as showing different clinical and pathological features. ${ }^{27-30}$ The lysine at codon 219 is believed to be protective against SCJD. Only one of the present P102L GSS case was E219K heterozygous; however, he displayed a SCJD phenotype with rapidly progressing dementia, PSWC in EEG, and a survival time of 10 months after the onset. Furthermore, this patient seemed to show less clinical symptoms than the other cases. More evidence is needed for exactly how the polymorphism at codon 219 influences on the clinical and pathological characteristics of P102L GSS in Chinese subjects.

In summary, based on data collected by the Chinese National Surveillance Network for human prion diseases from 2006 to 2018, P102L is the most-common GSS-associated mutation. More than half of the identified patients had the SCJD phenotype. Due to the complexity of clinical manifestations of GSS patients, PRNP sequencing is an indispensable tool for diagnosing GSS.

\section{Supplementary Materials}

The online-only Data Supplement is available with this article at https://doi.org/10.3988/jcn.2019.15.2.184.

\section{Conflicts of Interest}

The authors have no potential conflicts of interest to disclose.

\section{Acknowledgements}

We thank the referring patients and the family members for supplying the required information and donating specimens. This work was supported by National Science and Technology Major Project of the Ministry of Science and Technology of China (2017ZX10104001-002-005), Chinese National Natural Science Foundation Grants (81601810, 81630062, 81572048), Research project (2016YFC1202700) and SKLID Development Grant (2015SKLID503 and 2016SKLID603).

\section{REFERENCES}

1. Prusiner SB. Prions. Proc Natl Acad Sci U S A 1998;95:13363-13383.

2. Takada LT, Kim MO, Metcalf S, Gala II, Geschwind MD. Prion disease. Handb Clin Neurol 2018;148:441-464.

3. Kim MO, Takada LT, Wong K, Forner SA, Geschwind MD. Genetic PrP prion diseases. Cold Spring Harb Perspect Biol 2018;10:a033134.

4. Hainfellner JA, Brantner-Inthaler S, Cervenáková L, Brown P, Kitamoto T, Tateishi J, et al. The original Gerstmann-Sträussler-Scheinker family of Austria: divergent clinicopathological phenotypes but constant PrP genotype. Brain Pathol 1995;5:201-211.

5. Lloyd S, Mead S, Collinge J. Genetics of prion disease. Top Curr Chem 2011;305:1-22.

6. Doh-ura K, Tateishi J, Sasaki H, Kitamoto T, Sakaki Y. Pro $\rightarrow$ Leu change at position 102 of prion protein is the most common but not the sole mutation related to Gerstmann-Sträussler syndrome. Biochem Biophys Res Commun 1989;163:974-979.

7. Minikel EV, Vallabh SM, Lek M, Estrada K, Samocha KE, Sathirapongsasuti JF, et al. Quantifying prion disease penetrance using large population control cohorts. Sci Transl Med 2016;8:322ra9.

8. Hsiao K, Dlouhy SR, Farlow MR, Cass C, Da Costa M, Conneally $\mathrm{PM}$, et al. Mutant prion proteins in Gerstmann-Sträussler-Scheinker disease with neurofibrillary tangles. Nat Genet 1992;1:68-71.

9. Hsiao K, Baker HF, Crow TJ, Poulter M, Owen F, Terwilliger JD, et al. Linkage of a prion protein missense variant to Gerstmann-Sträussler syndrome. Nature 1989;338:342-345.

10. Takada LT, Kim MO, Cleveland RW, Wong K, Forner SA, Gala II, et al. Genetic prion disease: experience of a rapidly progressive dementia center in the United States and a review of the literature. Am J Med Genet B Neuropsychiatr Genet 2017;174:36-69.

11. Shi Q, Zhou W, Chen C, Zhang BY, Xiao K, Zhang XC, et al. The features of genetic prion diseases based on Chinese surveillance program. PLoS One 2015;10:e0139552.

12. Aguzzi A. Prion diseases of humans and farm animals: epidemiology, genetics, and pathogenesis. J Neurochem 2006;97:1726-1739.

13. Unterberger U, Voigtländer T, Budka H. Pathogenesis of prion diseases. Acta Neuropathol 2005;109:32-48.

14. Chen C, Dong XP. Epidemiological characteristics of human prion diseases. Infect Dis Poverty 2016;5:47.

15. Webb TE, Poulter M, Beck J, Uphill J, Adamson G, Campbell T, et al. Phenotypic heterogeneity and genetic modification of P102L inherited prion disease in an international series. Brain 2008;131:2632-2646.

16. Krasnianski A, Heinemann U, Ponto C, Kortt J, Kallenberg K, Varges $\mathrm{D}$, et al. Clinical findings and diagnosis in genetic prion diseases in Germany. Eur J Epidemiol 2016;31:187-196.

17. Higuma M, Sanjo N, Satoh K, Shiga Y, Sakai K, Nozaki I, et al. Relationships between clinicopathological features and cerebrospinal fluid biomarkers in Japanese patients with genetic prion diseases. PLoS One 2013;8:e60003.

18. Gao C, Shi Q, Tian C, Chen C, Han J, Zhou W, et al. The epidemiological, clinical, and laboratory features of sporadic Creutzfeldt-Jakob disease patients in China: surveillance data from 2006 to 2010. PLoS One 2011;6:e24231.

19. Zerr I, Kallenberg K, Summers DM, Romero C, Taratuto A, Heinemann $\mathrm{U}$, et al. Updated clinical diagnostic criteria for sporadic Creutzfeldt-Jakob disease. Brain 2009;132:2659-2668.

20. Shi Q, Zhou W, Chen C, Gao C, Xiao K, Wang J, et al. Quality evaluation for the surveillance system of human prion diseases in China 
based on the data from 2010 to 2016. Prion 2016;10:484-491.

21. Geschwind MD. Prion diseases. Continuum (Minneap Minn) 2015; 21:1612-1638.

22. Vitali P, Maccagnano E, Caverzasi E, Henry RG, Haman A, TorresChae C, et al. Diffusion-weighted MRI hyperintensity patterns differentiate CJD from other rapid dementias. Neurology 2011;76:1711-1719.

23. Kovács GG, Puopolo M, Ladogana A, Pocchiari M, Budka H, Van Duijn C, et al. Genetic prion disease: the EUROCJD experience. Hum Genet 2005;118:166-174.

24. Mead S, Uphill J, Beck J, Poulter M, Campbell T, Lowe J, et al. Genome-wide association study in multiple human prion diseases suggests genetic risk factors additional to PRNP. Hum Mol Genet 2012;21: 1897-1906.

25. Lloyd SE, Mead S, Collinge J. Genetics of prion diseases. Curr Opin Genet Dev 2013;23:345-351.

26. Jeong BH, Nam JH, Lee YJ, Lee KH, Jang MK, Carp RI, et al. Poly- morphisms of the prion protein gene (PRNP) in a Korean population. J Hum Genet 2004;49:319-324.

27. Bishop MT, Pennington C, Heath CA, Will RG, Knight RS. PRNP variation in UK sporadic and variant Creutzfeldt Jakob disease highlights genetic risk factors and a novel non-synonymous polymorphism. BMC Med Genet 2009;10:146.

28. Tanaka Y, Minematsu K, Moriyasu H, Yamaguchi T, Yutani C, Kitamoto T, et al. A Japanese family with a variant of Gerstmann-SträusslerScheinker disease. J Neurol Neurosurg Psychiatry 1997;62:454-457.

29. Lee SM, Ju YR, Choi BY, Hyeon JW, Park JS, Kim CK, et al. Genotype patterns and characteristics of PRNP in the Korean population. Prion 2012;6:375-382.

30. Furukawa H, Kitamoto T, Tanaka Y, Tateishi J. New variant prion protein in a Japanese family with Gerstmann-Sträussler syndrome. Brain Res Mol Brain Res 1995;30:385-388. 